\title{
Protocol for the systematic review of the prevention, treatment and public health management of impetigo, scabies and fungal skin infections in resource-limited settings
}

Philippa May ${ }^{1 *}$ (D) Asha Bowen ${ }^{2}$, Steven Tong ${ }^{3}$, Andrew Steer ${ }^{4}$, Sam Prince ${ }^{5}$, Ross Andrews ${ }^{3}$, Bart Currie $^{3}$ and Jonathan Carapetis ${ }^{2}$

\begin{abstract}
Background: Impetigo, scabies, and fungal skin infections disproportionately affect populations in resource-limited settings. Evidence for standard treatment of skin infections predominantly stem from hospital-based studies in high-income countries. The evidence for treatment in resource-limited settings is less clear, as studies in these populations may lack randomisation and control groups for cultural, ethical or economic reasons. Likewise, a synthesis of the evidence for public health control within endemic populations is also lacking. We propose a systematic review of the evidence for the prevention, treatment and public health management of skin infections in resource-limited settings, to inform the development of guidelines for the standardised and streamlined clinical and public health management of skin infections in endemic populations.

Methods: The protocol has been designed in line with the Preferred Reporting Items for Systematic Reviews and Meta-Analyses Protocols statement. All trial designs and analytical observational study designs will be eligible for inclusion. A systematic search of the peer-reviewed literature will include PubMed, Excertpa Medica and Global Health. Grey literature databases will also be systematically searched, and clinical trials registries scanned for future relevant studies. The primary outcome of interest will be the clinical cure or decrease in prevalence of impetigo, scabies, crusted scabies, tinea capitis, tinea corporis or tinea unguium. Two independent reviewers will perform eligibility assessment and data extraction using standardised electronic forms. Risk of bias assessment will be undertaken by two independent reviewers according to the Cochrane Risk of Bias tool. Data will be tabulated and narratively synthesised. We expect there will be insufficient data to conduct meta-analysis. The final body of evidence will be reported against the Grades of Recommendation, Assessment, Development and Evaluation grading system.

(Continued on next page)
\end{abstract}

\footnotetext{
* Correspondence: Philippa.may@telethonkids.org.au

${ }^{1}$ Wesfarmers Centre for Vaccines and Infectious Diseases, Telethon Kids

Institute, University of Western Australia, PO Box 855, West Perth, WA 6872,

Australia

Full list of author information is available at the end of the article
} 
(Continued from previous page)

Discussion: The evidence derived from the systematic review will be used to inform the development of guidelines for the management of skin infections in resource-limited settings. The evidence derived will be intended for use by clinicians, public health practitioners and policy makers in the treatment of skin infections and the development of skin infection control programmes. The review will identify any gaps in the current evidence to provide direction for future research.

Systematic review registration: PROSPERO CRD42015029453

Keywords: Impetigo, Scabies, Tinea, Therapeutics, Public health, Endemic diseases

\section{Introduction}

The skin infections impetigo, scabies and tinea disproportionately affect the most vulnerable populations [1-4]. Children in low-income countries, tropical regions and disadvantaged populations within high-income countries are amongst the worst affected [5-7]. Impetigo and scabies are correlated; people with scabies are at risk of secondary bacterial infection with impetigo and areas with a high prevalence of one condition often have a high prevalence of the other [5, 8]. Tinea infections (superficial mycoses) are also common in tropical and resource-limited settings and occur more frequently in children than in adult populations $[9,10]$. Tinea may also be complicated by impetiginisation [9]. Without treatment of impetigo or secondarily infected dermatoses, serious and sometimes fatal complications can occur, including sepsis, invasive infection and poststreptococcal sequelae $[4,11-16]$.

\section{Impetigo}

Impetigo is characterised by golden exudate and crust, typically caused by the bacteria Staphylococcus aureus, the prevalent bacteria in temperate regions, or Streptococcus pyogenes (group A beta haemolytic Streptococcus (GAS)), the predominant organism in tropical contexts $[6,17]$. Impetigo can occur on any part of the body where breaks in skin integrity have occurred [18-20]. Infestation with the scabies mite is a major contributing factor in resourcelimited settings and tropical regions $[6,12,21]$. The global paediatric population suffering from impetigo at any one time has been estimated at $>162$ million; but this affliction is not uniformly distributed, with children in tropical regions and resource-limited settings carrying the greatest burden of disease [22].

The medical treatment of impetigo comprises either topical or systemic antibiotics directed at the two common pathogens [23-26]. Hand hygiene [27], topical disinfectants $[24,25]$ and school exclusion measures $[28,29]$ may be used as adjuncts for treatment and contribute to disease control. Natural therapies and traditional medicines such as tea tree oil [30] and cocky apple tree poultices or suspensions [31] are used by some populations.
Koning et al. concluded that the topical antibiotics, fusidin and mupirocin, were the most effective treatment for mild to moderate impetigo [24, 25]. However, the majority of studies included in the review were conducted in high-income countries and hospital outpatient settings [24, 25]. In contrast, in Australian Aboriginal and Torres Strait Islander communities, the recommended treatment for impetigo has been intramuscular benzathine penicillin, based on non-randomised trials conducted over 40 years ago [32, 33]. Although Koning et al. did not limit the Cochrane systematic review by population and setting [24, 25], few studies were included that involved populations in resource-limited settings, as studies conducted in these settings may have been non-randomised and uncontrolled and thus did not satisfy inclusion criteria. As such, it is questionable whether the findings, namely the first-line use of topical antibiotics, can be recommended for treatment of impetigo in resource-limited settings, particularly as impetigo is often severe in these settings [21]. With recent evidence showing the efficacy of oral trimethoprimsulfamethoxazole [21], growing concern regarding antimicrobial resistance to topical agents [34] and rising rates of methicillin-resistant S. aureus (MRSA) [35], a review of the evidence base for effective treatment of impetigo in resource-limited settings is needed. In addition, primordial prevention interventions for impetigo targeting the social determinants of health, which underlie the burden of skin infections across the world $[1-3,36]$, require review.

\section{Scabies}

Scabies, due to infestation by the mite Sarcoptes scabiei var. hominis [4], is a human only skin disease characterised by intense itch and cutaneous eruption [37, 38]. Scratching due to scabies interrupts the usual skin barrier [37], facilitating the entry of bacteria, causing secondary bacterial infection and its subsequent sequelae $[37,38]$. Scabies mites crawl, but do not fly or jump; thus transmission occurs through skin-to-skin contact and occasionally through fomite spread $[39,40]$ as the mite survives for several days off the human host [40]. 
The estimated global prevalence of scabies in 2010 was over 100 million people [41]. A recent systematic review reported the highest point prevalence of paediatric scabies in the resource-limited settings of Panama (78 \% of children under age 2 years affected), Fiji ( $44 \%$ of 5 - to 9 -yearolds affected) and Australia, where one third of Aboriginal children in remote communities are affected [42].

Clinical treatment of scabies is with topical or oral anti-parasitic agents [38, 43]. Herbal therapies and traditional medicines, including natural pyrethrins, tea tree oil [44] and emu bush [45], may also be effective, as well as culturally acceptable in certain settings. Additional methods for the prevention of transmission of scabies include barrier precautions, environmental decontamination measures [40] and multifaceted population-level approaches in endemic communities involving community education, community involvement and treatment of cases and contacts [46].

In the original Cochrane Review in 2000, Walker and Johnstone concluded that topical permethrin was superior to all other treatments for scabies [43]. In the 2007 update, oral ivermectin was supported as an effective treatment, although was not licensed for use in the treatment of scabies in many countries, and topical permethrin remained superior to oral ivermectin [38]. This systematic review needs updating to take into account the recently published mass drug administration (MDA) experimental studies [16, 47].

Beyond specific clinical treatment, a Cochrane review of measures to reduce the transmission of scabies was unable to provide recommendations due to a lack of RCTs examining these [40]. A systematic review of population-level approaches to scabies prevention and control is needed but challenging, due to lack of randomisation or comparisons of programmatic approaches to scabies management in endemic communities [48]. However, effective programmes have been delivered in Australian Aboriginal communities $[49,50]$. As described by Wong et al., a programme delivered in a large Aboriginal community in the Top End of the Northern Territory involving extensive community support, education, screening of school children, MDA of permethrin and environmental health services led to a significant reduction in scabies and pyoderma prevalence at 7 [50] and 15 months [49]. As scabies is endemic in areas where overcrowding, social disruption and poverty exist [51, 52], a synthesis of the best available evidence for scabies control that determines treatment effectiveness, prevention of re-infection and cross-infection within families and communities in resource-limited settings is required [37, 43, 53].

\section{Crusted scabies}

Whilst classical scabies infestations involve 5 to 15 scabies mites [37, 39], people with crusted (or Norwegian) scabies have hyperinfestation with thousands to millions of mites and are highly infectious [37, 38]. There is a spectrum of severity, from mild cases to severe hyperkeratotic dermatosis [37]. The crusted scabies burden in remote Aboriginal communities of the Northern Territory in Australia has been estimated at 2.4 per 1000 population (Personal communication, S. Prince 2016). The global burden of crusted scabies is yet to be described.

Management of crusted scabies frequently involves hospitalisation of the affected individual to prevent community spread, provide frequent topical and systemic scabicides along with application of keratolytics to manage hyperkeratosis $[46,54,55]$. Environmental measures are recommended to decontaminate the environment, where the heavy infestation of scabies mites can survive for several days in the absence of the human host, including washing of clothing and bed linen [37]. In addition, household spraying or fogging with insecticides has been recommended in some clinical guidelines to prevent transmission via fomites $[56,57]$.

Individuals with crusted scabies are core transmitters of scabies within households or communities [37, 47]. Thus, public health interventions that identify and treat people with crusted scabies in communities where scabies is endemic are likely to reduce the burden of scabies [53, 58]. However, social stigma associated with scabies, particularly crusted scabies, is significant and must be approached with respect and sensitivity [37, 58]. Community-based identification and management of individuals with crusted scabies within the privacy of their homes has been effective in the Northern Territory of Australia using a chronic disease model of care [58]. The high transmissibility of crusted scabies from an index case [40, 47] has implications for scabies control in communities where individuals with crusted scabies reside $[47,58]$; hence, a review of the evidence for effective and socially acceptable methods of crusted scabies management and control is needed.

\section{Tinea/ringworm}

Tinea infections are classified according to the site of the human body affected [9]. Tinea corporis of the body, tinea capitis of the scalp and tinea unguium [onychomycosis] of the nail are caused by infection with dermatophytes of the genera Epidermophyton, Microsporum and Trichophyton [10, 59].

Between 10 and $20 \%$ of the world's population are affected by fungal skin infections [10]. Estimates of the disease burden have generally focussed on the prevalence of causative organisms, rather than the clinical disease entities [60, 61]. However, the World Health Organization (WHO) has estimated that 7-33\% of children assessed in clinical surveys in developing countries are affected by tinea capitis [17], and a recent 
systematic review reported the global prevalence of onychomycosis as $11 \%$ [62].

Clinical treatment for tinea infection includes oral and topical antifungals and adjunctive treatment, such as selenium sulphide shampoo [7, 9, 59]. Laser therapy for tinea unguium is emerging [63-65] but its applicability in resource-limited settings remains unknown.

Tinea is highly communicable, even amongst asymptomatic carriers [9] with community-wide implications for control programmes [17]. Identification of infected contacts is important to prevent recontamination and dissemination [17]. Additional methods for tinea control include washing and disinfection of clothing, towels, combs and brushes; discouraging sharing of these items amongst household members; and concurrent treatment of family members $[9,17]$.

\section{Rationale for a systematic review applicable to resource- limited settings}

People living in regions with endemic rates of scabies and impetigo $[5,66,67]$ are at substantial risk of infective complications, post-streptococcal sequelae and physical disability [68, 69]. A coordinated and evidence-based approach to treatment and population management is needed [17]. All Cochrane systematic reviews for the treatment of impetigo, scabies and tinea relate to participants in highincome countries, hospital settings and people with higher socioeconomic status $[10,24,25,38,40]$. As such, there is no current evidence-based consensus on the management of skin infections found in the peer-reviewed literature to support clinical and public health decision making in endemic settings. We aim to develop guidelines for the prevention, treatment and community control of impetigo, scabies and fungal infections in resource-limited settings, as the predominant skin infections in these populations $[1,17]$, drivers of high demand for health care $[17,28,66]$ and potential precursors to rare but serious illness $[13,17]$. To develop a robust guideline for common skin infections in endemic populations, a peer-reviewed, systematic review of the evidence base applicable to resource-limited settings is required. Due to the overlap between the occurrence of impetigo, scabies and fungal infections $[8,9]$ and the need for streamlined guidance on the management of these common conditions in resource-limited settings [17], a broad systematic review encompassing multiple conditions will be conducted. The objective of the review is to assess the evidence for treatment and public health management of impetigo, scabies, crusted scabies, tinea capitis, tinea corporis and tinea unguium in endemic settings.

\section{Methods}

This protocol is in accordance with the Preferred Reporting items for Systematic Reviews and Meta-Analyses-
Protocol (PRISMA-P) statement (Additional file 1) [70]. The systematic review will be conducted using standard systematic review methodology based on the Cochrane handbook and reported in accordance with the PRISMA statement $[71,72]$. The review will be overseen by a steering group, comprised of experts in the field. The review is registered with PROSPERO (International prospective register of systematic reviews) at the National Institute for Health Research and Centre for Reviews and Dissemination (CRD) at the University of York (registration number CRD42015029453).

\section{Eligibility criteria \\ Types of participants and setting}

The review will consider studies that include participants of any age, gender or country of origin within resourcelimited settings, tropical regions, remote Aboriginal and Torres Strait Islander communities and resource-limited populations in Organisation for Economic Co-operation and Development (OECD) countries or low, low-middle and middle income countries. The review will include people who have been diagnosed with impetigo, scabies, crusted scabies, tinea capitis, tinea corporis or tinea unguium.

\section{Intervention}

The review will examine any clinical or public health interventions that aim to reduce skin infections. Treatment interventions may include either pharmaceutical or complementary medicines, including traditional management of skin infections in relevant to the setting. Public health interventions may include communicable disease control, primary health care delivery, environmental health, health promotion, education or community-level interventions.

\section{Comparator}

Studies with any type of comparator will be included.

\section{Outcomes and prioritisation}

The primary outcomes will include cure (i.e. resolution) or improvement in the condition. A decrease in prevalence will be a primary outcome for population-based studies. Secondary outcomes to be extracted will include the clearance of organism on microbiological testing, relief of symptoms, recurrence rate, adherence to treatment or management regimen, patient acceptability, adverse effects or the proportion of contacts diagnosed with the condition within 8 weeks of diagnosis of the index case [40].

\section{Study design}

Any experimental study designs will be eligible for inclusion, including RCTs, quasi-experimental, before and after 
studies and cross over trials. The review will also include cohort and case-control analytical study designs.

\section{Information sources}

Online databases of the peer- reviewed literature including PubMed, Excerpta Medica Database (EMBASE) and Global Health will be searched. In addition, grey literature will be accessed via the Australian Institute of Health and Welfare (AIHW), Australian Indigenous HealthInfoNet, Informit, OaIster databases and World Health Organization (WHO) website. In addition, clinical trial registries ANZ Clinical Trials Registry, ClinicalTrials.gov and WHO International Clinical Trials Registry will be searched to identify any relevant studies underway. The reference lists of all included full-text articles will be handsearched for relevant titles for inclusion.

\section{Search strategy}

The search strategy aims to identify all relevant treatments and public health interventions applied within a resource-limited context regardless of publication status (published, unpublished, in press or in progress). The search strategy will use a combination of keywords and subject headings relating to the setting and outcomes of interest. A sample search strategy for PubMed is included (Additional file 2). Terms will be adapted according to the unique search features of the respective databases. The search will be restricted to studies published since 1960 for feasibility. Any study published in English will be considered for inclusion in the review. Non-English language articles will be excluded due to resource constraints.

\section{Study records}

\section{Data management}

Titles and abstracts will be retrieved and screened using Endnote reference management software. Full-text screening and data extraction will be managed using the web-based software platform Covidence (Veritas Health Innovation, Melbourne, VIC, Australia).

\section{Selection process}

Two independent reviewers will screen titles, and then abstracts, for suitability using the index terms. Following this, the full text of potentially relevant reports will be retrieved where available. Full-text reports will be assessed by two independent reviewers for compliance with eligibility criteria. Reviewers will be assigned to a subset of papers after determining that they are not either an author of the paper or involved in data collection for the study. Where possible, we will attempt to correspond with primary authors via email when eligibility for inclusion is unclear. Any disagreements that arise between the reviewers regarding the studies for final inclusion will be resolved via a consensus decision of the review steering group. Members of the review steering group who have authored a paper will be excluded from any consensus decisions in relation to a paper they have authored.

\section{Data collection process}

Data will be extracted using standardised forms by two independent reviewers. Any disagreements will be resolved by consensus amongst reviewers or will be referred to the review steering group.

\section{Data items}

Data to be extracted from included studies comprises the following: author, journal, study year or where missing year of publication, funding source, study aims, setting, study design, participants, intervention type, frequency, dose, duration, co-intervention, primary outcomes, secondary outcomes and results (including effect estimates). Details of programme evaluation will be extracted if available.

\section{Risk of bias assessment}

An assessment of methodological quality by the two independent reviewers performing data extraction on a study will be based on The Cochrane Collaboration's tool for assessing risk of bias [72]. For RCTs, this will comprise the following: random sequence generation; allocation concealment; blinding of participants, personnel and outcome assessors; incomplete outcome data; reporting bias and other sources of bias that may have affected the results. As recommended by Higgins and Greene, non-RCT experimental and controlled studies will also use the domains based on The Cochrane Collaboration's tool for assessing risk of bias [72]. The "other sources of bias" domain will include an assessment for confounding for non-RCTs. For observational studies, random sequence generation and allocation concealment will not be applicable, but other domains of blinding, incomplete outcome data, selective outcome reporting and other sources of bias will be assessed, which will include an assessment of identified and other potential confounders. Risk of bias assessments will subsequently be rated as high, low or unclear in a "Risk of bias" table. Any disagreements that arise will be resolved via a consensus decision of the review steering group.

\section{Data synthesis}

We expect there to be insufficient data to conduct metaanalyses. Quantitative findings will be presented in narrative form including additional technical tables and figures to aid in data interpretation where appropriate. Where possible, opinion-based findings will be synthesised and presented in a "Characteristics of included 
information" tables or text. Results for each condition will be presented in turn.

A single, comprehensive set of synthesised quantitative findings will be presented in a "Summary of Findings" table for each condition from which to develop guidelines. The quality of the evidence will be rated using the Grading of Recommendations, Assessment, Development and Evaluation (GRADE) grading system [73].

\section{Meta-biases}

Studies will be included only once (i.e. multiple reports of data from the same study will be screened by author names, setting, institution, type of intervention and date and duration of the study) to reduce the risk of publication bias. We will assess for evidence of selective outcome reporting when the outcomes are described in the "Methods" section but not reported in the "Results" section of the same study report.

\section{Confidence in cumulative evidence}

We will assess the underlying reasons for any discrepancies in results between studies, taking into account study design characteristics and risk of bias. The strength of the overall body of evidence will be reported against the Grading of Recommendations, Assessment, Development and Evaluation (GRADE) grading system [73].

\section{Discussion}

This review will provide a synthesis of the existing literature applicable to resource-limited settings, with GRADE evidence gradings [73], to inform the development of guidelines for the management of skin infections in endemic populations and particularly Australian Aboriginal and Torres Strait Islander communities. Recommendations for skin infection prevention, treatment, public health management and programme evaluation will be the focus of the guideline. A limitation of the review is that the methodology (i.e. the inclusion of non-randomised trials and observational studies across multiple conditions) will likely preclude formulation of overall effect estimates via meta-analysis due to the expected high heterogeneity between studies. Any evidence gaps that are exposed will highlight areas for future research. A summarised version of the review will be submitted for publication. The guidelines and summary systematic review paper will be made available on the Internet for use by clinicians and public health practitioners. In addition, policymakers and health organisations may choose to use the evidence base as a resource for the development of local treatment protocols and skin health programmes.

\section{Additional files}

Additional file 1: PRISMA-P (Preferred Reporting Items for Systematic review and Meta-Analysis Protocols) 2015 checklist: recommended items to address in a systematic review protocol. (DOC 86 kb)

Additional file 2: PubMed Search Strategy. (DOCX 26 kb)

\section{Abbreviations}

AlHW: Australian Institute of Health and Welfare; ANZCTR: Australian New Zealand Clinical Trials Registry; EMBASE: Excerpta Medica Database; GAS: Group A Streptococcus; GRADE: Grades of Recommendation, Assessment, Development and Evaluation; MDA: Mass drug administration; MRSA: Methicillin-resistant Staphylococcus aureus; OECD: Organisation for Economic Co-operation and Development; PRISMA-P: Preferred Reporting items for Systematic Reviews and Meta-Analyses-Protocol; WHO: World Health Organization

\section{Acknowledgements}

Not applicable.

\section{Funding}

The authors have not received any external funding in support of this protocol. Three of the authors receive scholarships from the National Health and Medical Research Council of Australia: ST and AS receive Career Development Fellowships and $A B$ receives an Early Career Fellowship. The National Health and Medical Research Council has not had any role in the design of the protocol or writing of the manuscript and will not have any role in data extraction, analysis, interpretation of data or reporting of the systematic review.

\section{Availability of data and materials}

Not applicable.

\section{Authors' contributions}

$P M$ and $A B$ wrote the protocol. $A B$ and $J C$ conceived the initial idea for the review. JC, ST, AS, SP, RA and BC critically appraised the protocol and contributed to its design and development. All authors approved the final version and take responsibility for its content.

Authors' information

Not applicable.

Competing interests

The authors declare that they have no competing interests.

Consent for publication

Not applicable.

Ethics approval and consent to participate

Not applicable.

\section{Author details}

${ }^{1}$ Wesfarmers Centre for Vaccines and Infectious Diseases, Telethon Kids Institute, University of Western Australia, PO Box 855, West Perth, WA 6872, Australia. ${ }^{2}$ Telethon Kids Institute, University of Western Australia, West Perth, Australia. ${ }^{3}$ Menzies School of Health Research, Charles Darwin University, Casuarina, Australia. ${ }^{4}$ Murdoch Children's Research Institute, University of Melbourne, Parkville, Australia. ${ }^{5}$ One Disease, Tiwi, Australia.

Received: 15 June 2016 Accepted: 9 September 2016 Published online: 23 September 2016

\section{References}

1. Hay RJ, Johns NE, Williams HC, Bolliger IW, Dellavalle RP, Margolis DJ, et al. The global burden of skin disease in 2010: an analysis of the prevalence and impact of skin conditions. J Clin Investig Dermatol. 2014;134(6):1527-34.

2. Heukelbach J, Mazigo HD, Ugbomoiko US. Impact of scabies in resource-poor communities. Curr Opin Infect Dis. 2013;26(2):127-32.

3. Kline K, McCarthy JS, Pearson M, Loukas A, Hotez PJ. Neglected tropical diseases of Oceania: review of their prevalence, distribution, and opportunities for control. PLoS Negl Trop Dis. 2013;7(1), e1755. 
4. Engelman D, Kiang K, Chosidow O, McCarthy J, Fuller C, Lammie P, et al. Toward the global control of human scabies: introducing the International Alliance for the Control of Scabies. PLoS Negl Trop Dis. 2013;7(8), e2167.

5. Carapetis JR, Steer AC, Mulholland EK, Weber M. The global burden of group A streptococcal diseases. Lancet Infect Dis. 2005;5(11):685-94.

6. Steer AC, Jenney AWJ, Kado J, Batzloff MR, La Vincente S, Waqatakirewa L, et al. High burden of impetigo and scabies in a tropical country. Lancet Infect Dis. 2009;3(6), e467.

7. Andrews RM, McCarthy J, Carapetis JR, Currie BJ. Skin disorders, including pyoderma, scabies, and tinea infections. Pediatr Clin North Am. 2009;56(6):1421-40

8. Romani L, Koroivueta J, Steer AC, Kama M, Kaldor JM, Wand H, et al. Scabies and impetigo prevalence and risk factors in Fiji: a national survey. PLoS Negl Trop Dis. 2015;9(3), e0003452.

9. Hawkins DM, Smidt AC. Superficial fungal infections in children. Pediatr Clin North Am. 2014;61(2):443-55.

10. El-Gohary M, van Zuuren EJ, Fedorowicz Z, Burgess H, Doney L, Stuart B, et al. Topical antifungal treatments for tinea cruris and tinea corporis. Cochrane Database Syst Rev. 2014;8:Cd009992. doi:10.1002/14651858.CD009992.

11. Cunningham MW. Pathogenesis of group A streptococcal infections. Clin Microbiol Rev. 2000;13(3):470-511.

12. Steer A, Danchin M, Carapetis J. Group A streptococcal infections in children. J Paediatr Child Health. 2007:43(4):203-13.

13. Walker MJ, Barnett TC, MCArthur JD, Cole JN, Gillen CM, Henningham A, et al. Disease manifestations and pathogenic mechanisms of group A streptococcus. Clin Microbiol Rev. 2014;27(2):264-301.

14. Baldwin DS, Gluck MC, Schacht RG, Gallo G. The long-term course of poststreptococcal glomerulonephritis. Ann Intern Med. 1974;80(3):342-58.

15. Hoy WE, White AV, Dowling A, Sharma SK, Bloomfield H, Tipiloura BT, et al. Post-streptococcal glomerulonephritis is a strong risk factor for chronic kidney disease in later life. Kidney Int. 2012;81(10):1026-32.

16. Romani L, Whitfeld MJ, Koroivueta J, Kama M, Wand H, Tikoduadua L, et al. Mass drug administration for scabies control in a population with endemic disease. N Engl J Med. 2015;373(24):2305-13.

17. Mahé AH, Hay R. Epidemiology and management of common skin diseases in children in developing countries. Geneva: World Health Organization; 2005.

18. Ferrieri P, Dajani AS, Wannamaker LW, Chapman SS. Natural history of impetigo. I. Site sequence of acquisition and familial patterns of spread of cutaneous streptococci. J Clin Invest. 1972;51(11):2851-62.

19. Harambat J, van Stralen KJ, Kim JJ, Tizard EJ. Epidemiology of chronic kidney disease in children. Pediatr Nephrol. 2012;27(3):363-73.

20. Kakar N, Kumar V, Mehta G, Sharma RC, Koranne RV. Clinico-bacteriological study of pyodermas in children. J Dermatol. 1999;26(5):288-93.

21. Bowen AC, Tong SY, Andrews RM, O'Meara IM, McDonald MI, Chatfield $M D$, et al. Short-course oral co-trimoxazole versus intramuscular benzathine benzylpenicillin for impetigo in a highly endemic region: an open-label, randomised, controlled, non-inferiority trial. Lancet. 2014, 384(9960):2132-40.

22. Bowen AC, Mahe A, Hay RJ, Andrews RM, Steer AC, Tong SY, et al. The global epidemiology of impetigo: a systematic review of the population prevalence of impetigo and pyoderma. PLoS One. 2015;10(8), e0136789.

23. George A, Rubin G. A systematic review and meta-analysis of treatments for impetigo. Br J Gen Pract. 2003;53(491):480-7.

24. Koning $S$, van der Sande R, Verhagen AP, van Suijlekom-Smit LW, Morris AD, Butler CC, et al. Interventions for impetigo. Cochrane Database Syst Rev. 2012;1:Cd003261.

25. Koning S, Verhagen AP, van Suijlekom-Smit LW, Morris A, Butler CC, van der Wouden JC. Interventions for impetigo. Cochrane Database Syst Rev. 2004;2004(2):Cd003261

26. Hartman-Adams H, Banvard C, Juckett G. Impetigo: diagnosis and treatment. Am Fam Physician. 2014;90(4):229-35.

27. Luby SP, Agboatwalla M, Feikin DR, Painter J, Billhimer W, Altaf A, et al Effect of handwashing on child health: a randomised controlled trial. Lancet. 2005:366(9481):225-33.

28. Department of Health. Communicable Disease Guidelines: For teachers, child care workers, local government authorities and medical practitioners. 2014 ed. Government of Western Australia. 2013. http://www.public.health. wa.gov.au/cproot/471/2/2014_communicable_disease_guidelines_web.pdf. Accessed 15 Jun 2016

29. National Health and Medical Research Council. Staying healthy: Preventing infectious diseases in early childhood education and care services (updated
June 2013). 5th ed. NHMRC. 2012. https://www.nhmrc.gov.au/_files_nhmrc/ publications/attachments/ch55_staying_healthy_5th_edition_150602.pdf. Accessed 15 Jun 2016

30. Martin KW, Ernst E. Herbal medicines for treatment of bacterial infections: a review of controlled clinical trials. J Antimicrob Chemother. 2003;51(2):241-6.

31. Williams C. Medicinal Plants in Australia: Volume 4 - An Antipodean Apothecary. Dural: Rosenberg Publishing; 2013.

32. Esterly NB, Markowitz M. The treatment of pyoderma in children. JAMA. 1970;212(10):1667-70.

33. Dillon Jr HC. The treatment of streptococcal skin infections. J Pediatr. 1970;76(5):676-84.

34. Williamson DA, Monecke S, Heffernan H, Ritchie SR, Roberts SA, Upton A, et al. High usage of topical fusidic acid and rapid clonal expansion of fusidic acid-resistant Staphylococcus aureus: a cautionary tale. Clin Infect Dis. 2014:59(10):1451-4.

35. Tong SY, Varrone L, Chatfield MD, Beaman M, Giffard PM. Progressive increase in community-associated methicillin-resistant Staphylococcus aureus in Indigenous populations in northern Australia from 1993 to 2012 Epidemiol Infect. 2015;143(7):1519-23.

36. Kearns T, Clucas D, Connors C, Currie BJ, Carapetis JR, Andrews RM. Clinic attendances during the first 12 months of life for Aboriginal children in five remote communities of northern Australia. PLoS One. 2013;8(3), e58231.

37. Heukelbach J, Feldmeier H. Scabies. Lancet. 2006;367(9524):1767-74.

38. Strong M, Johnstone P. Interventions for treating scabies. Cochrane Database Syst Rev. 2007;18(3):Cd000320.

39. Chosidow O. Scabies and pediculosis. Lancet. 2000;355(9206):819-26.

40. FitzGerald D, Grainger RJ, Reid A. Interventions for preventing the spread of infestation in close contacts of people with scabies. Cochrane Database Syst Rev. 2014:(2):Cd009943

41. Vos T, Flaxman AD, Naghavi M, Lozano R, Michaud C, Ezzati M, et al. Years lived with disability (YLDs) for 1160 sequelae of 289 diseases and injuries 1990-2010: a systematic analysis for the Global Burden of Disease Study 2010. Lancet. 2012;380(9859):2163-96.

42. Romani L, Steer AC, Whitfeld MJ, Kaldor JM. Prevalence of scabies and impetigo worldwide: a systematic review. Lancet Infect Dis. 2015.

43. Walker GJ, Johnstone PW. Interventions for treating scabies. Cochrane Database Syst Rev. 2000;24(3):Cd000320.

44. Thomas J, Carson CF, Peterson GM, Walton SF, Hammer KA, Naunton M, et al. Therapeutic potential of tea tree oil for scabies. Am J Trop Med Hyg. 2016;94(2):258-66

45. Richmond G. A review of the use of Eremophila (Myoporaceae) by Australian Aborigines. J Adelaide Bot Gard. 1993;15:101-7.

46. Currie BJ, McCarthy JS. Permethrin and ivermectin for scabies. N Engl J Med. 2010;362(8):717-25.

47. Kearns TM, Speare R, Cheng AC, McCarthy J, Carapetis JR, Holt DC, et al. Impact of an ivermectin mass drug administration on scabies prevalence in a remote Australian aboriginal community. PLoS Negl Trop Dis. 2015;9(10), e0004151.

48. Haar K, Romani L, Filimone R, Kishore K, Tuicakau M, Koroivueta J, et al. Scabies community prevalence and mass drug administration in two Fijian villages. Int J Dermatol. 2014;53(6):739-45.

49. Wong LC, Amega B, Barker R, Connors C, Dulla ME, Ninnal A, et al. Factors supporting sustainability of a community-based scabies control program. Australas J Dermatol. 2002:43(4):274-7.

50. Wong LC, Amega B, Connors C, Barker R, Dulla ME, Ninnal A, et al. Outcome of an interventional program for scabies in an Indigenous community. Med J Aust. 2001;175(7):367-70.

51. Feldmeier $H$, Jackson A, Ariza $L$, Calheiros CM, Soares Vde $L$, Oliveira FA, et al. The epidemiology of scabies in an impoverished community in rural Brazil: presence and severity of disease are associated with poor living conditions and illiteracy. J Am Acad Dermatol. 2009;60(3):436-43.

52. Worth C, Heukelbach J, Fengler G, Walter B, Liesenfeld O, Hengge U, et al. Acute morbidity associated with scabies and other ectoparasitoses rapidly improves after treatment with ivermectin. Pediatr Dermatol. 2012;29(4):430-6.

53. La Vincente S, Kearns T, Connors C, Cameron S, Carapetis J, Andrews R. Community management of endemic scabies in remote aboriginal communities of northern Australia: low treatment uptake and high ongoing acquisition. PLoS Negl Trop Dis. 2009;3(5), e444.

54. Monsel G, Chosidow O. Management of scabies. Skin Therapy Lett. 2012;17(3):1-4. 
55. Hay RJ, Steer AC, Engelman D, Walton S. Scabies in the developing world-its prevalence, complications, and management. Clin Microbiol Infect. 2012;18(4):313-23.

56. Centre for Disease Control. Healthy Skin Program: Guidelines for Community Control of Scabies, Skin Sores and Crusted Scabies in the Northern Territory. 3rd ed. Department of Health, Northern Territory. 2015. http://www.health.nt. gov.au/library/scripts/objectifyMedia.aspx?file=pdf/10/83.pdf\&sitelD=1\&str_ title=Healthy\%20Skin\%20Program.pdf. Accessed 15 Jun 2016.

57. One Disease. Managing Crusted Scabies in Remote Aboriginal Communities. One Disease. 2014. http://static1.squarespace.com/static/ 56b141b11 bbee06392b16f36/t/56f3152340261ddd9bc22453/1458771247857/ Crusted+Scabies+guide+2014_final.pdf. Accessed 15 Jun 2016.

58. Lokuge B, Kopczynski A, Woltmann A, Alvoen F, Connors C, Guyula T, et al. Crusted scabies in remote Australia, a new way forward: lessons and outcomes from the East Arnhem Scabies Control Program. Med J Aust. 2014;200(11):644-8.

59. Hay R. Superficial fungal infections. Medicine. 2013;41(12):716-8.

60. Seebacher C, Bouchara JP, Mignon B. Updates on the epidemiology of dermatophyte infections. Mycopathologia. 2008;166(5-6):335-52.

61. Fuller LC. Changing face of tinea capitis in Europe. Curr Opin Infect Dis. 2009;22(2):115-8.

62. Sigurgeirsson B, Baran R. The prevalence of onychomycosis in the global population: a literature study. J Eur Acad Dermatol Venereol. 2014;28(11):1480-91.

63. Lim E, Kim H, Park Y, Lee Y, Seo Y, Kim C, et al. Toenail onychomycosis treated with a fractional carbon-dioxide laser and topical antifungal cream. J Eur Acad Dermatol Venereol. 2014;70(5):918-23.

64. Zhang RN, Wang DK, Zhuo FL, Duan XH, Zhang XY, Zhao JY. Longpulse Nd:YAG 1064-nm laser treatment for onychomycosis. Chin Med J. 2012;125(18):3288-91.

65. Xu X, Lai A, Zheng J, Li J. Therapeutic effect of $1064 \mathrm{~nm}$ Nd:YAG laser on 32 onychomycosis cases. China Trop Med. 2014;14(10):1246-8.

66. Clucas DB, Carville KS, Connors C, Currie BJ, Carapetis JR, Andrews RM. Disease burden and health-care clinic attendances for young children in remote aboriginal communities of northern Australia. Bull World Health Organ. 2008;86(4):275-81.

67. McMeniman E, Holden L, Kearns T, Clucas DB, Carapetis JR, Currie BJ, et al. Skin disease in the first two years of life in Aboriginal children in East Arnhem Land. Australas J Dermatol. 2011;52(4):270-3.

68. Carapetis JR, Walker AM, Hibble M, Sriprakash KS, Currie BJ. Clinical and epidemiological features of group A streptococcal bacteraemia in a region with hyperendemic superficial streptococcal infection. Epidemiol Infect. 1999;122(1):59-65.

69. Marshall CS, Cheng AC, Markey PG, Towers RJ, Richardson LJ, Fagan PK, et al. Acute post-streptococcal glomerulonephritis in the Northern Territory of Australia: a review of 16 years data and comparison with the literature. Am J Trop Med Hyg. 2011;85(4):703-10.

70. Moher D, Shamseer L, Clarke M, Ghersi D, Liberati A, Petticrew M, et al. Preferred reporting items for systematic review and meta-analysis protocols (PRISMA-P) 2015 statement. Syst Rev. 2015;4:1.

71. Moher D, Liberati A, Tetzlaff J, Altman DG. Preferred reporting items for systematic reviews and meta-analyses: the PRISMA statement. Int I Surg. 2010;8(5):336-41.

72. Higgins JPT, Green S. Cochrane Handbook for Systematic Reviews of Interventions. Version 5.1.0 (updated March 2011). The Cochrane Collaboration. 2011. http://handbook.cochrane.org/ Accessed 15 Jun 2016

73. Grade Working Group. Grading quality of evidence and strength of recommendations. BMJ. 2004:328(7454):1490.

\section{Submit your next manuscript to BioMed Central and we will help you at every step:}

- We accept pre-submission inquiries

- Our selector tool helps you to find the most relevant journal

- We provide round the clock customer support

- Convenient online submission

- Thorough peer review

- Inclusion in PubMed and all major indexing services

- Maximum visibility for your research

Submit your manuscript at www.biomedcentral.com/submit
C Biomed Central 